\title{
Implementation and Performance Evaluation of Overlay End System Multicast (ESM) for Stable and Fast Streaming of Multimedia Applications over Satellite Networks
}

\section{Khaled Elleithy, Thomas Arciuolo and Akram Abu-aisheh}

Department of Computer Engineering, College of Engineering, Business, and Education University of Bridgeport, Bridgeport, Connecticut, USA

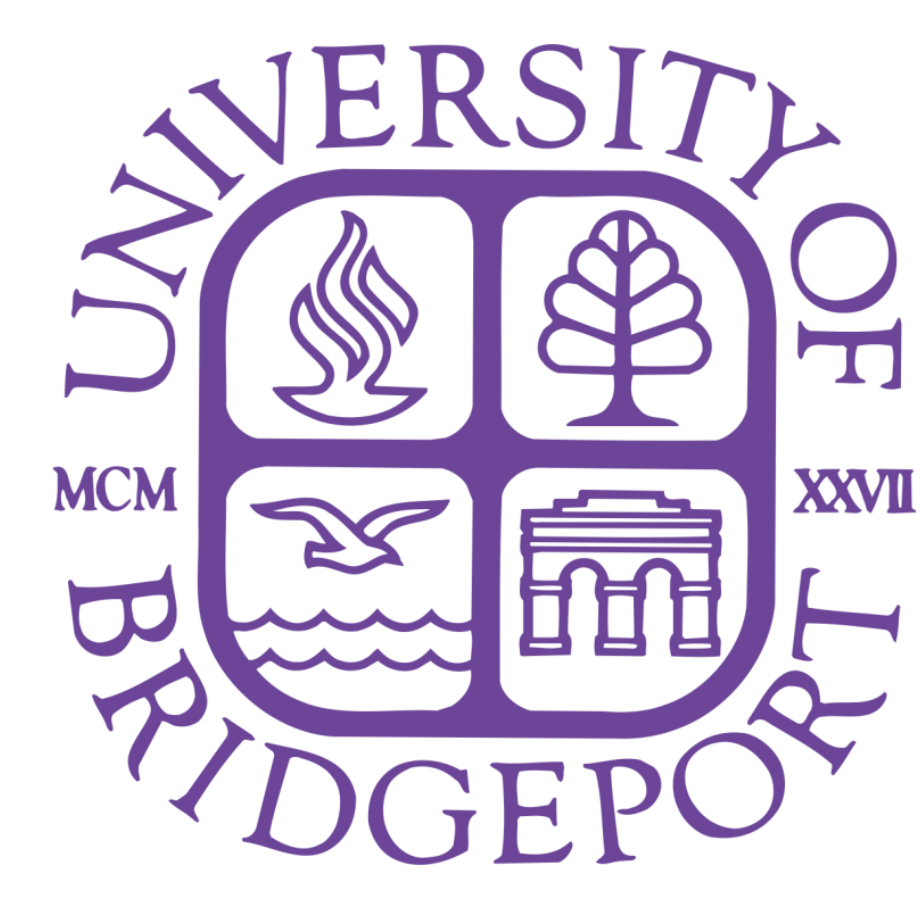

\section{Abstract}

Our paper investigates the issues related to the implementation of an Overlay End-System Multicast to support satellite Internet and networks. Furthermore, we develop both analytical and mathematical models to evaluate the performance of overlay networks for stable and fast streaming of multimedia applications over satellite networks. The results produced by this research would help satellite networks designers to design and implement more robust and efficient future networks that support a variety of multimedia applications.

\section{Proposed Solution}

Testing was done in 4 different configurations:

- 13 Virtual Satellites

- 7 Virtual Satellites

- 1 Virtual Satellite

- 0 Virtual Satellites (Ground Stations 1 and 2 only)
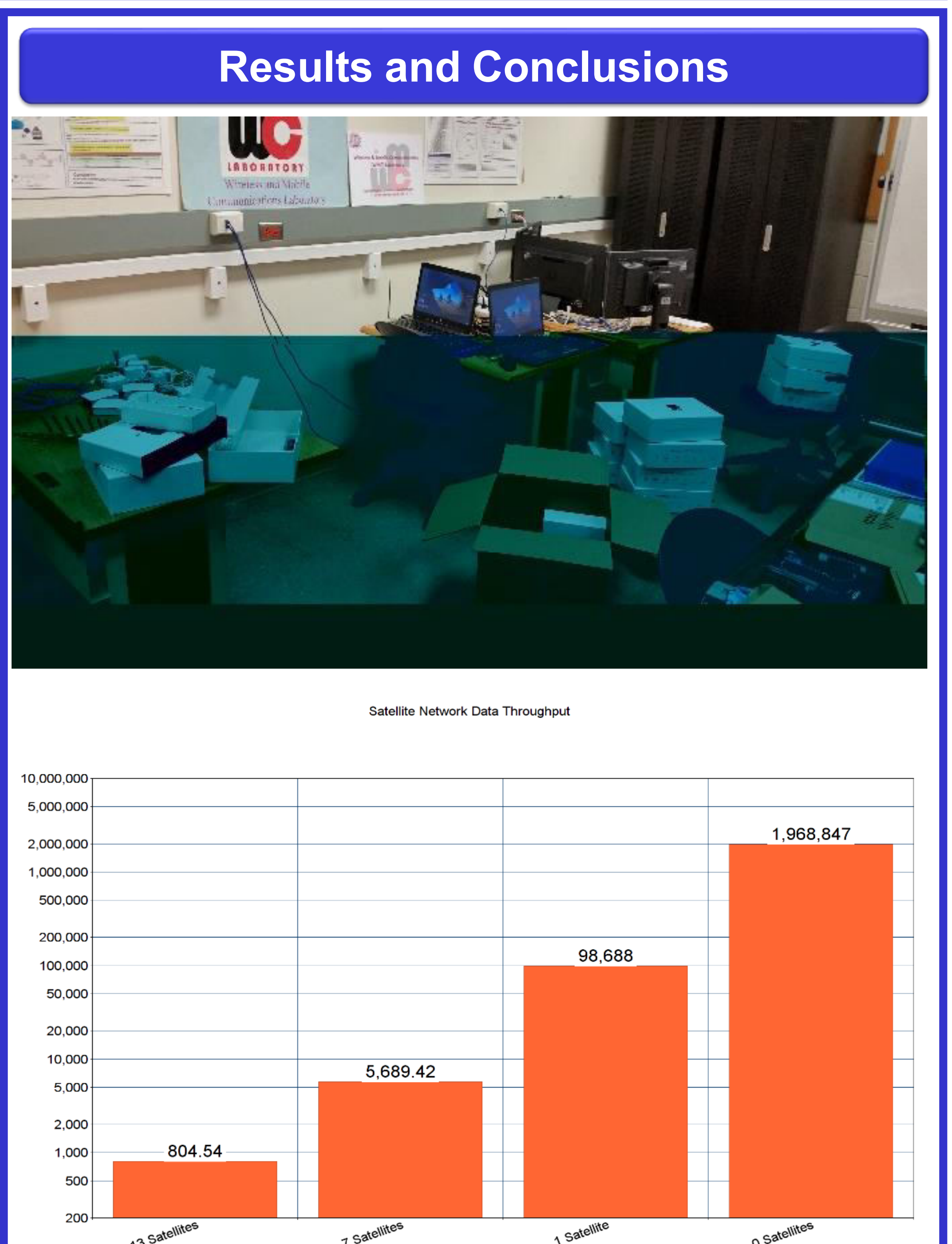

$y=-20286.73216 \times 3+427245.1768 \times 2-2277117.445 x+1968847 ;$ Error $=0.0$

$x=$ Number of Virtual Satellites, $y=$ Average Data Throughput (bytes)

The Satellite Network Data Throughput versus the Number of Satellites.

The varying Modulation type, Baud Rates, and Message Length were found to have minimal impact on the Virtual Satellite Network's overall performance in terms of data throughput, reliability, or performance. The most significant overall performance variations occurred with changes in the number of satellites in the communications chain between Ground Station 1 and Ground Station 2.

\section{Introduction}

The IP protocol faces many problems related to scalability, network management, deployment, and support for higher-layer functionality. Overlay End-System Multicast systems (ESM) has received attention in the literature due to their capabilities to potentially address most problems associated with IP multicast. Recent research results discussed satellite networks' limitations to support high volume traffic and suggested some partial solutions. The limitations of these solutions are related to Multimedia applications over satellite networks. Multimedia applications would likely form a significant fraction of satellite traffic between space stations and Satellite Internet soon. Concerning the current transmission rates over the satellite Internet, it is highly likely that the multimedia applications would quickly surpass other data transmission forms in terms of its client base's size shortly. An example of such usage is shown in the Figure for integrated and interoperable satellite and terrestrial networks. The research aims to improve the satellite Internet and the satellite networks that support Multimedia applications. This research's objectives are very relevant and extremely important to NASA's strategic goal 4: Optimize Capabilities and Operations, specifically, "Provide access to space for the Nation's civil sector satellite and robotic planetary missions".

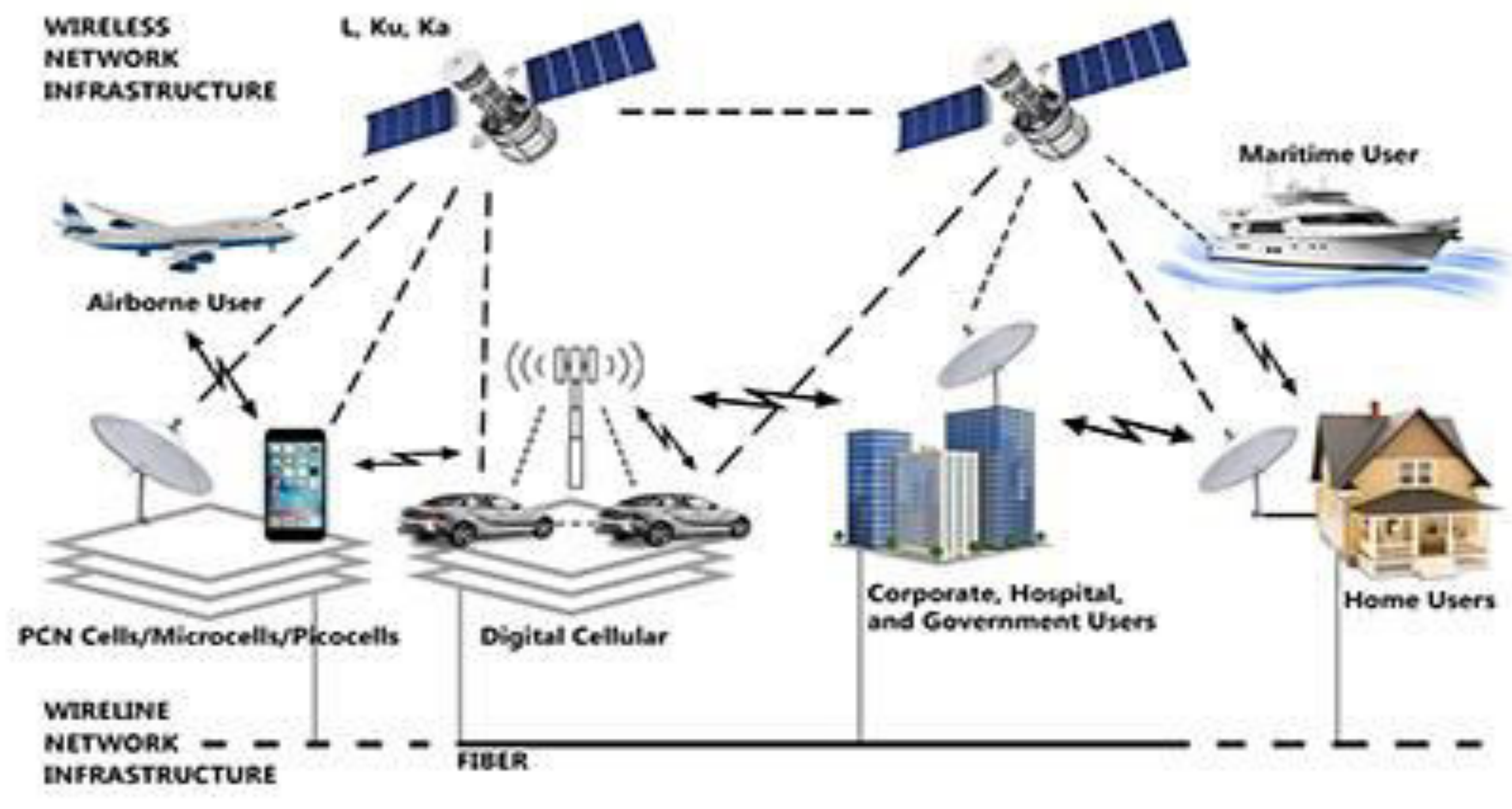

Laboratory Virtual Satellite Network Experiments
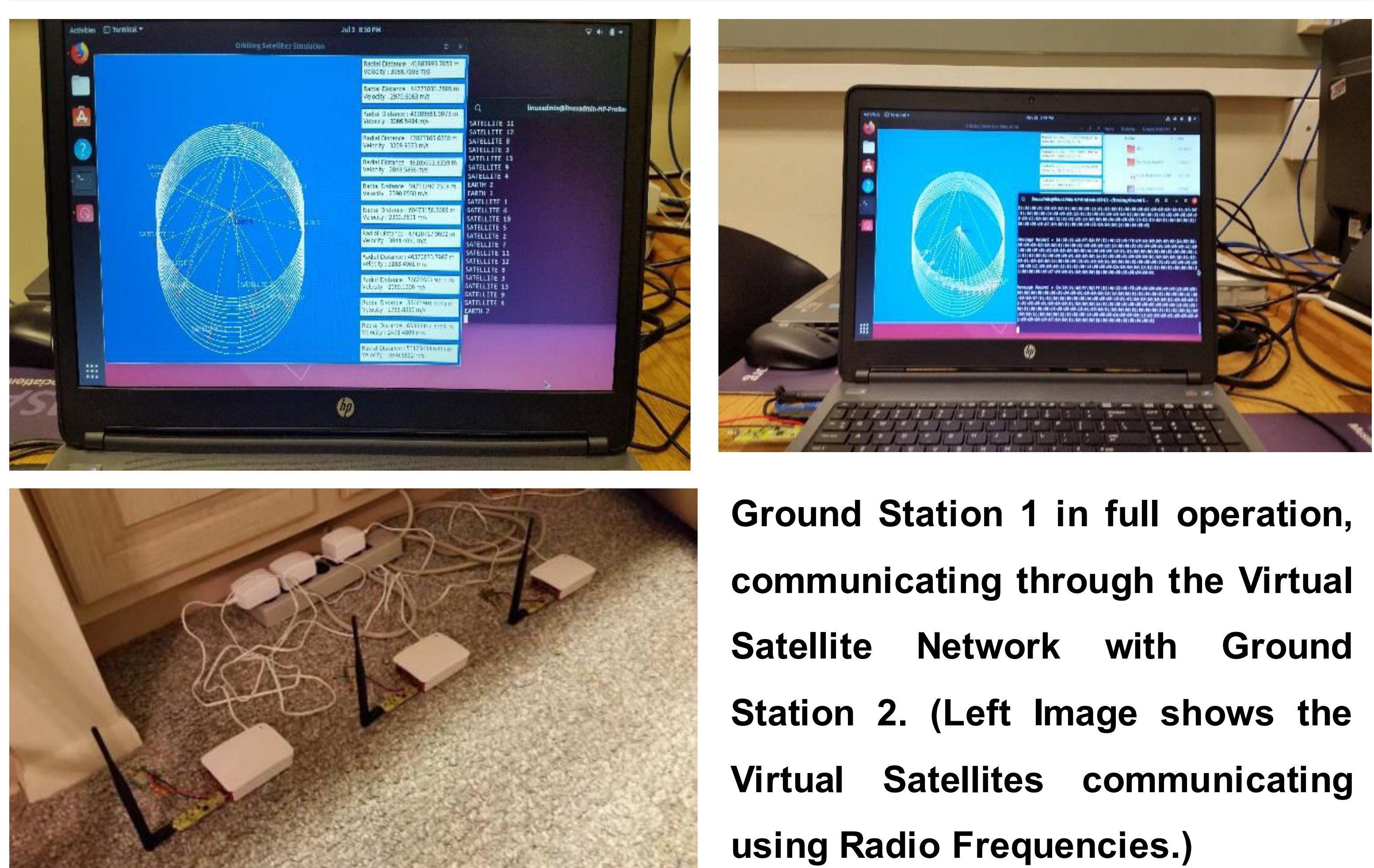

Ground Station 1 in full operation, communicating through the Virtual Satellite Network with Ground Station 2. (Left Image shows the Virtual Satellites communicating using Radio Frequencies.)

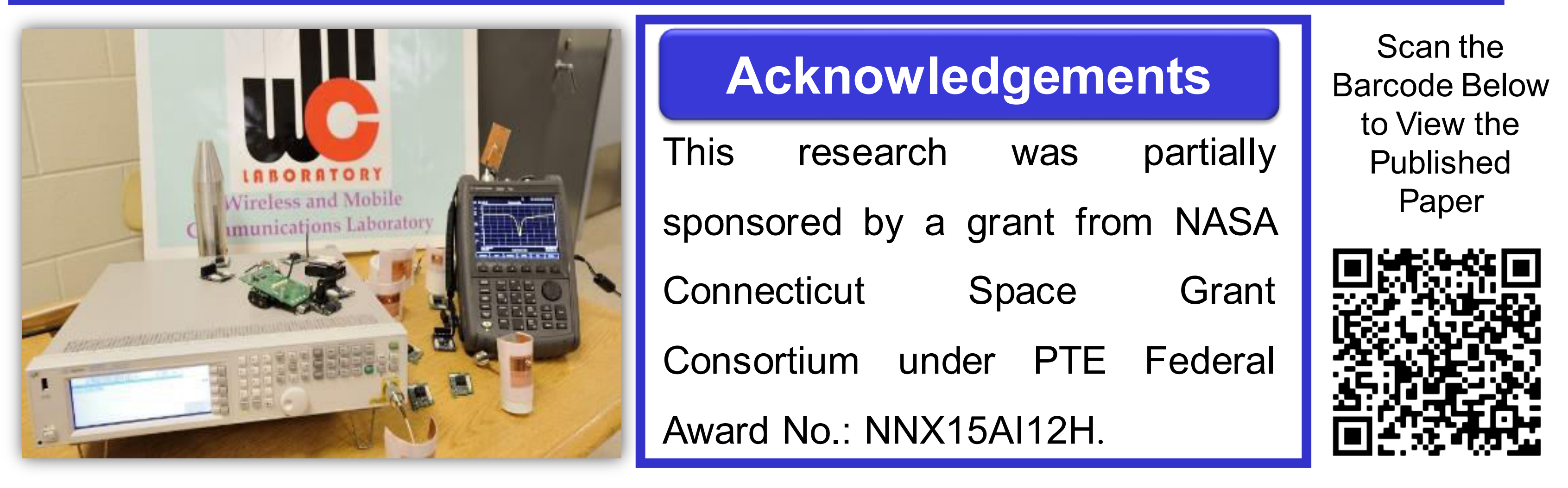

